Pacific Journal of Mathematic 


\title{
CONJUGATE SPACE REPRESENTATIONS OF BANACH SPACES
}

\author{
EMILE BOYd ROTH
}

Let a linear homeomorphism $T$ from a Banach space $X$ onto the conjugate space $Y^{*}$ of a Banach space $Y$ be called a conjugate space representation of $X$. If $T: X \rightarrow Y^{*}$ and $U: X \rightarrow Z^{*}$ are two conjugate space representations of $X$, say that $T$ and $U$ are essentially different if there is no linear homeomorphism $P$ from $Y$ onto $Z$ satisfying $P^{*}=T \circ U^{-1}$. It is proven here that if a nonreflexive Banach space has one conjugate space representation, it has uncountably many essentially different conjugate space representations. A Banach space $X$ with norm $p$ will be denoted by $(X, p)$ when it is important to emphasize the norm. The dual of $p$ is the norm $p^{*}$ defined on the conjugate space $(X, p)^{*}$ of $(X, p)$ by

$$
p^{*}(f)=\sup \{|f(x)|: x \in X \text { and } p(x)=1\} \text {. }
$$

It is proven here that if $T:(X, p) \rightarrow(Y, r)^{*}$ and $U:(X, p) \rightarrow$ $(Z, s)^{*}$ are two essentially different conjugate space representations of $(X, p)$, then there exists a norm $q$ on $X$ equivalent to $p$ such that $q \circ T^{-1}=r_{1}^{*}$ for some norm $r_{1}$ on $Y$ equivalent to $r$, but such that $q \circ U^{-1} \neq s_{1}^{*}$ for any norm $s_{1}$ on $Z$ equivalent to $s$.

Williams has shown [7, Th. 1, p. 163] that a Banach space $(X, p)$ is reflexive if and only if every norm $q$ on $X^{*}$ equivalent to $p^{*}$ is the dual of some norm on $X$ equivalent to $p$. We show here that if $(X, p)$ is a nonreflexive Banach space, then there exists a norm $q$ on $X^{*}$ equivalent to $p^{*}$ such that $q$ is not the dual of any norm on $X$ equivalent to $p$, but such that the Banach space $\left(X^{*}, q\right)$ is isometrically isomorphic to a conjugate Banach space. By contrast, Klee [3, Th. 4, p. 21] has exhibited a Banach space $(X, p)$ and a norm $q$ on $X^{*}$ equivalent to $p^{*}$ such that $\left(X^{*}, q\right)$ is not isometrically isomorphic to a conjugate Banach space.

We shall use the following notation. If $A$ and $B$ are sets, $A \backslash B$ denotes the set of elements in $A$ but not in $B$. If $x$ is an element in a linear space, $[x]$ denotes the linear span of $x$. If $A$ and $B$ are linear subspaces of a linear space $X$, and if $A \cap B=\{0\}$, then $A \oplus B$ denotes the linear direct sum of $A$ and $B$. If $A$ is a subset of a normed linear space $(X, p), A^{\perp}$ donotes the annihilator of $A$ in $X^{*}$. If $A$ is a subset of the conjugate space $X^{*}$ of a normed linear space $(X, p), A_{\perp}$ denotes the set of elements in $X$ annihilated by $A$. If $(X, p)$ is a normed linear space, $J_{X}$ denotes the canonical map from $X$ into $X^{* *}$ defined by 


$$
\left(J_{X} x\right) f=f(x) \text { for all } x \in X \text { and } f \in X^{*} \text {. }
$$

Lemma. If $T: X \rightarrow Y^{*}$ and $U: X \rightarrow Z^{*}$ are two conjugate space representations of a Banach space $X$, then $T$ and $U$ are essentially different if and only if

$$
T^{*}\left[J_{Y} Y\right] \neq U^{*}\left[J_{Z} Z\right] .
$$

Proof. (i) Suppose $T$ and $U$ are not essentially different. Then there exists a linear homeomorphism $P$ from $Y$ onto $Z$ satisfying $P^{*}=$ $T \circ U^{-1}$. It is straightforward to verify that $\left(P^{* * *}\left(J_{Y} y\right)\right) g=\left(J_{Z}(P y)\right) g$ for all $y \in Y$ and $g \in Z^{*}$; that is, $P^{* *} \circ J_{Y}=J_{Z} \circ P$. Therefore $T^{*}\left[J_{Y} Y\right]=$ $\left(P^{*} \circ U\right)^{*}\left[J_{Y} Y\right]=\left(U^{*} \circ P^{* *} \circ J_{Y}\right)[Y]=U^{*}\left[J_{Z} Z\right]$.

(ii) Suppose $T^{*}\left[J_{Y} Y\right]=U^{*}\left[J_{Z} Z\right]$. Let $P=J_{Z}^{-1} \circ U^{*-1} \circ T^{*} \circ J_{Y}=$ $J_{Z}^{-1} \circ\left(T \circ U^{-1}\right)^{*} \circ J_{Y}$. Then $P$ is a linear homeomorphism from $Y$ onto $Z$. It can be verified directly that $\left(P^{*} g\right) y=\left(\left(T \circ U^{-1}\right) g\right) y$ for all $g \in Z^{*}$ and $y \in Y$. Therefore $P^{*}=T \circ U^{-1}$.

THeorem 1. Suppose that $(X, p)$ is a nonreflexive Banach space which is linearly homeomorphic to the conjugate $\left(Y^{*}, r^{*}\right)$ of a Banach space $(Y, r)$. Then there exists an uncountable collection of essentially different conjugate space representations $U_{\alpha}:(X, p) \rightarrow\left(Z_{\alpha}^{*}, s_{\alpha}^{*}\right)$ such that each space $\left(Z_{\alpha}, s_{\alpha}\right)$ is linearly homeomorphic to $(Y, r)$.

Proof. By hypothesis there exists a linear homeomorphism $T$ from $(X, p)$ onto $\left(Y^{*}, r^{*}\right)$. Let $M=T^{*}\left[J_{Y} Y\right]$. Then [4, p. 577] $M$ is a minimal total norm-closed subspace of $\left(X^{*}, p^{*}\right)$. That is, $M$ is total and norm-closed, and no proper subspace of $M$ is both total and normclosed. If $L$ is any norm-closed subspace of $X^{*}$, let $Q_{L}$ denote the canonical map from $X$ into $L^{*}$ defined by

$$
\left(Q_{L} x\right) f=f(x) \text { for all } x \in X \text { and } f \in L .
$$

In particular, $Q_{X^{*}}$ is the canonical map $J_{X}$ from $X$ into $X^{* *}$. By [4, p. 577], the map $Q_{L}$ is a linear homeomorphism from $(X, p)$ onto $\left(L^{*},\left(p^{*} \mid L\right)^{*}\right)$ if and only if $L$ is a minimal total norm-closed subspace of $\left(X^{*}, p^{*}\right)$. Since $(X, p)$ is not reflexive, $Q_{X^{*}}$ is not a linear homeomorphism from $(X, p)$ onto $\left(X^{* *}, p^{* *}\right)$, and $X^{*}$ is not a minimal total norm-closed subspace of $X^{*}$.

Let $f \in X^{*}$. Let us show that there is a minimal total norm-closed subspace $B$ of $X^{*}$ such that $f \in B$ and such that $B$ is linearly homeomorphic to $(Y, r)$. If $f \in M$, we may take $B=M$. Now suppose $f \notin M$. By a theorem of Dixmier [1, Th. 11, p. 1065] a norm-closed total subspace $V$ of the conjugate $E^{*}$ of a Banach space $E$ is a minimal total norm-closed subspace of $E^{*}$ if and only if $E^{* *}=J_{E} E \oplus V^{\perp}$. Thus 
we have $X^{* *}=J_{X} X \oplus M^{\perp}$. Let $H=[f]^{\perp}$. By the Hahn-Banach Theorem [6, Corollary 2, p. 67], $H \nsupseteq M^{\perp}$ so $J_{X} X \oplus\left(H \cap M^{\perp}\right)$ is a maximal subspace of $X^{* *}$. Now $H \nsupseteq J_{X} X$ since $f \neq 0$, so $J_{X} X \oplus\left(H \cap M^{\perp}\right) \neq H$. Since $J_{X} X \oplus\left(H \cap M^{\perp}\right)$ and $H$ are distinct maximal subspaces of $X^{* *}$, there exists $G \in H$ such that $X^{* *}=J_{X} X \oplus\left(H \cap M^{\perp}\right) \oplus[G]$. Let $D=$ $\left(H \cap M^{\perp}\right) \oplus[G]$, and let $B=D_{\perp}$. Then [6, $(x)$, p. 238] $B$ is a normclosed subspace of $\left(X^{*}, p^{*}\right)$. The subspaces $H$ and $M^{\perp}$ are $w\left(X^{* *}, X^{*}\right)$ closed [6, (x), p. 238], so $H \cap M^{\perp}$ is $w\left(X^{* *}, X^{*}\right)$-closed. Therefore [6, Corollary 5, p. 192] $D$ is $w\left(X^{* *}, X^{*}\right)$ closed, and [6, Th. 1, p. 238] $B^{\perp}=$ $\left(D_{\perp}\right)^{\perp}=D$. Now $B$ is a total subspace of $X^{*}$ since $B^{\perp} \cap J_{X} X=\{0\}$. By the theorem of Dixmier mentioned above, $B$ is a minimal total norm-closed subspace of $X^{*}$. By the Hahn-Banach Theorem, we have $f \in B$ since $B^{\perp} \subseteq H=[f]^{\perp}$. Now observe that both $B$ and $M$ are maximal subspaces of $\left(H \cap M^{\perp}\right)_{\perp}$, and consequently each of them is linearly homeomorphic to the topological direct sum of $B \cap M$ with a onedimensional space. Therefore $B$ is linearly homeomorphic to $M$ which is in turn linearly homeomorphic to $Y$.

Let $\left\{Z_{\alpha}: \alpha \in \Phi\right\}$ be the collection of all minimal total norm-closed subspaces of $X^{*}$ which are linearly homeomorphic to $Y$. For each $\alpha \in \Phi$ let $s_{\alpha}=p^{*} \mid Z_{\alpha}$. We have established that every element $f \in X^{*}$ is contained in some $Z_{\alpha}$. Each $Z_{\alpha}$ is nowhere dense in $X^{*}$ since each $Z_{\alpha}$ is a proper norm-closed subspace of $X^{*}$. Since $X^{*}$ is a complete linear metric space, the Baire Category Theorem guarantees that $X^{*}$ is not a countable union of nowhere dense sets. Therefore $\left\{Z_{\alpha}: \alpha \in \Phi\right\}$ is an uncountable collection. For every $\alpha \in \Phi$, let $U_{\alpha}=Q_{z_{\alpha}}$. It is straightforward to verify that $U_{\alpha}^{*} \circ J_{Z_{\alpha}}$ is the identity map on $Z_{\alpha}$. Thus $U_{\alpha}^{*}\left[J_{Z_{\alpha}} Z_{\alpha}\right]=Z_{\alpha}$. Therefore by the lemma $U_{\beta}$ and $U_{\alpha}$ are essentially different whenever $\beta, \alpha \in \Phi$ and $\beta \neq \alpha$.

Theorem 2. Suppose that $T:(X, p) \rightarrow\left(Y^{*}, r^{*}\right)$ and $U:(X, p) \rightarrow$ $\left(Z^{*}, s^{*}\right)$ are two essentially different conjugate space representations. Then there exists a norm $q$ on $X$ equivalent to $p$ such that $q \circ T^{-1}$ is the dual of some norm $r_{1}$ on $Y$ equivalent to $r$, but such that $q \circ U^{-1}$ is not the dual of any norm $s_{1}$ on $Z$ equivalent to $s$.

Remark. An interesting example may be obtained by letting $X$, $Y$ and $Z$ be the sequence spaces $l, c$, and $c_{0}$, respectively.

Proof of Theorem 2. Let $A=T^{*}\left[J_{Y} Y\right]$ and let $B=U^{*}\left[J_{Z} Z\right]$. Then $A \neq B$ by the lemma. By [4, p. 577], $A$ and $B$ are minimal total norm-closed subspaces of $X^{*}$. The map $T$ is a vector space isomorphism from $X$ onto $Y^{*}$ and $T^{*}\left[J_{Y} Y\right]=A$; it follows that $T$ is a $w(X, A)-w\left(Y^{*}, J_{Y} Y\right)$-homeomorphism. Let $S=T^{-1}\left[\left\{g \in Y^{*}: r^{*}(g) \leqq 1\right\}\right]$. Then $S$ is $w(X, A)$-compact, because $\left\{g \in Y^{*}: r^{*}(g) \leqq 1\right\}$ is $w\left(Y^{*}, J_{Y} Y\right)$ - 
compact by the Banach-Alaoglu Theorem [6, Th. 1, p. 239]. Since $A \neq B$ and since $A$ and $B$ are both minimal with respect to certain properties, we must have $A \nsubseteq B$. Thus there exists $f \in A \backslash B$. Let $L=f^{-1}(0)$ and let $V=L \cap S$. The subspace $L$ is $w(X, A)$-closed [6, Th. 3, p. 186] since $f \in A$. Thus $V$ is $w(X, A)$-compact.

Now $f$ is not $w(X, B)$-continuous [2, Th. 9, p. 421] since $f \notin B$, so [6, Th. 3, p. 186] $L$ is not $w(X, B)$-closed. However, $L$ is norm-closed [6, Th. 3, p. 186] since $f \in X^{*}$. Thus $U[L]$ is a norm-closed subspace of $\left(Z^{*}, s^{*}\right)$, but $U[L]$ is not $w\left(Z^{*}, J_{Z} Z\right)$-closed. Let $K$ be the $w(X, B)$ closure of $V$. Then [2, Lemma 4, p. 415] $K$ is convex since $V$ is convex. Now $U[K]$ is a convex $w\left(Z^{*}, J_{Z} Z\right)$-closed subset of $Z^{*}$. By a corollary of the Krein-Šmulian Theorem [2, Corollary 9, p. 429], the linear span of a convex, weak* closed set is weak* closed if and only if it is norm-closed. Therefore $U[L] \neq \operatorname{span}(U[K])$. Consequently, $L \neq \operatorname{span}(K)$. However, $\operatorname{span}(K) \supseteqq \operatorname{span}(V)=L$, so there exists an element $x_{0} \in K \backslash L$. Let $W$ be the convex balanced hull of $V \cup\left\{(1 / 2) x_{0}\right\}$. Then if co denotes convex hull and bal denotes balanced hull, we have

$$
\begin{aligned}
W & =\operatorname{co}\left(\operatorname{bal}\left(V \cup\left\{\frac{1}{2} x_{0}\right\}\right)\right)=\operatorname{co}\left(\operatorname{bal}(V) \cup \operatorname{bal}\left\{\frac{1}{2} x_{0}\right\}\right) \\
& =\operatorname{co}\left(V \cup \text { bal }\left\{\frac{1}{2} x_{0}\right\}\right)
\end{aligned}
$$

which is $w(X, A)$-closed [2, Lemma 5, p. 415] since the sets $V$ and bal $\left\{(1 / 2) x_{0}\right\}$ are convex and $w(X, A)$-compact. The set $W$ is norm-closed since the norm topology is stronger than the $w(X, A)$ topology. Also $W$ is norm-bounded since $V$ and bal $\left\{(1 / 2) x_{0}\right\}$ are norm-bounded. Now span $(W)=X$ since span $(W)$ properly contains the maximal subspace $L$. Thus for any $x \in X$ there exist elements $w_{1}, \cdots w_{N} \in W$ and nonzero numbers $t_{1}, \cdots, t_{N}$ such that $x=t_{1} w_{1}+\cdots+t_{N} w_{N}$. Let $t=\sum_{i=1}^{N}\left|t_{i}\right|$. Then $x / t \in W$ since $W$ is convex and balanced. Thus $W$ is absorbing. We have shown that $W$ is a convex, balanced, absorbing, norm-closed norm-bounded subset of the Banach space $(X, p)$. Therefore $W$ is a normneighborhood of zero since Banach spaces are barrelled. By [6, p. 58] the gauge $q$ of $W$ is a norm on $X$ equivalent to $p$, and $W=$ $\{x \in X: q(x) \leqq 1\}$.

Let $q_{1}=q \circ T^{-1}$. Then $q_{1}$ is a norm on $Y^{*}$ equivalent to $r^{*}$ since $T$ is a linear $p-r^{*}$ homeomorphism. Also $\left\{g \in Y^{*}: q_{1}(g) \leqq 1\right\}=T[W]$, and $\left\{g \in Y^{*}: q_{1}(g) \leqq 1\right\}$ is $w\left(Y^{*}, J_{Y} Y\right)$-closed since $W$ is $w(X, A)$-closed. Singer has shown [5, Lemma 2, p. 450] that if $(E, h)$ is a Banach space, and if $h_{1}$ is a norm on $E^{*}$ equivalent to $h^{*}$, then $h_{1}$ is the dual of some norm on $E$ equivalent to $h$ if and only if the set $\left\{g \in E^{*}: h_{1}(g) \leqq 1\right\}$ is $w\left(E^{*}, J_{E} E\right)$-closed. (In one direction, of course, this is the well-known Banach-Alaoglu Theorem.) Therefore there exists a norm $r_{1}$ on $Y$ equivalent to $r$ such that $r_{1}^{*}=q_{1}=q \circ T^{-1}$. 
Let $q_{2}=q \circ U^{-1}$. Then $q_{2}$ is a norm on $Z^{*}$ equivalent to $s^{*}$ since $U$ is a linear $p-s^{*}$-homeomorphism. Also $\left\{g \in Z^{*}: q_{2}(g) \leqq 1\right\}=U[W]$. Now $x_{0} \notin W$, for if $x_{0}=c v+(1-c)(d)\left((1 / 2) x_{0}\right)$ with $v \in V, 0 \leqq c \leqq 1$, and $|d| \leqq 1$, then $(1-1 / 2(1-c) d) x_{0}=c v \in L$, so that $x_{0} \in L$, contrary to the definition of $x_{0}$. However, $x_{0}$ belongs to the $w(X, B)$-closure of $W$ since the $w(X, B)$-closure of $W$ contains the $w(X, B)$-closure of $V$, namely $K$. Therefore $W$ is not $w(X, B)$-closed. Thus $U[W]=$ $\left\{g \in Z^{*}: q_{2}(g) \leqq 1\right\}$ is not $w\left(Z, J_{Z} Z\right)$-closed. By the Banach-Alaoglu Theorem, there is no norm $s_{1}$ on $Z$ equivalent to $s$ such that $s_{1}^{*}=q_{2}=$ $q \circ U^{-1}$.

Corollary. If $(X, p)$ is a nonreflexive Banach space, there is a norm $q$ on $X^{*}$ equivalent to $p^{*}$ such that $q$ is not the dual of any norm on $X$ equivalent to $p$, but such that the Banach space $\left(X^{*}, q\right)$ is isometrically isomorphic to a conjugate Banach space.

Proof. Suppose that $(X, p)$ is a nonreflexive Banach space. By Theorem 1 there exists a conjugate space representation $T:\left(X^{*}, p^{*}\right) \rightarrow$ $\left(Y^{*}, r^{*}\right)$ such that $T$ is essentially different from the identity map $I$ on $X^{*}$. By Theorem 2 there exists a norm $q$ on $X^{*}$ equivalent to $p^{*}$ such that $q \circ T^{-1}=r_{1}^{*}$ for some norm $r_{1}$ on $Y$ equivalent to $r$, but such that $q \circ I^{-1}$ is not the dual of any norm on $X$ equivalent to $p$. Now $T$ is an isometric isomorphism from $\left(X^{*}, q\right)$ onto the conjugate Banach space $\left(Y^{*}, r_{1}^{*}\right)$.

\section{REFERENCES}

1. J. Dixmier, Sur un théoréme de Banach, Duke Math. J. 15 (1948), 1057-1071.

2. N. Dunford and J. T. Schwartz, Linear operators, Part I, Interscience Publishers, New York, 1958.

3. V. L. Klee, Jr., Some characterizations of reflexivity, Revista Ci., Lima 52 (1950), $15-23$.

4. A. F. Ruston, Conjugate Banach spaces, Proc. Cambridge Philos. Soc. 53 (1957), $576-580$.

5. I. Singer, On Banach spaces reflexive with respect to a linear subspace of their conjugate space, Bull. Math. Soc. Sci. Math. Phys. R. P. Roumaine (N. S.) (50) 2 (1958), 449-462.

6. A. Wilansky, Functional analysis, Blaisdell, New York, 1964.

7. J. P. Williams, A "metric" characterization of reflexivity, Proc. Amer. Math. Soc. 18 (1967), 163-165.

Received November 5, 1968. Most of the results of this paper are contained in the author's doctoral dissertation written at The Florida State University under the direction of Professor Ralph D. McWilliams.

The Florida State University 



\title{
PACIFIC JOURNAL OF MATHEMATICS
}

\author{
EDITORS
}

H. SAMELSON

Stanford University

Stanford, California 94305

J. DugunduI

Department of Mathematics

University of Southern California

Los Angeles, California 90007

RICHARD PIERCE

University of Washington

BASIL GORDON*

University of California

Seattle, Washington 98105

Los Angeles, California 90024

\section{ASSOCIATE EDITORS}

E. F. BeCKenbaCh

B. H. NeumanN

F. WOLF

K. YosHIDA

\section{SUPPORTING INSTITUTIONS}

\author{
UNIVERSITY OF BRITISH COLUMBIA \\ CALIFORNIA INSTITUTE OF TECHNOLOGY \\ UNIVERSITY OF CALIFORNIA \\ MONTANA STATE UNIVERSITY \\ UNIVERSITY OF NEVADA \\ NEW MEXICO STATE UNIVERSITY \\ OREGON STATE UNIVERSITY \\ UNIVERSITY OF OREGON \\ OSAKA UNIVERSITY \\ UNIVERSITY OF SOUTHERN CALIFORNIA
}

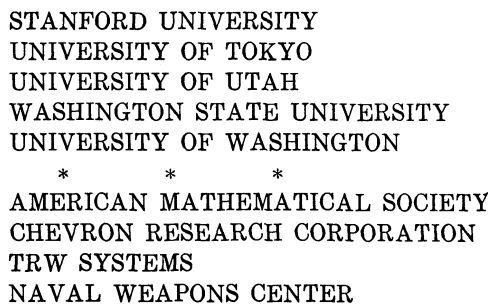

The Supporting Institutions listed above contribute to the cost of publication of this Journal, but they are not owners or publishers and have no responsibility for its content or policies.

Mathematical papers intended for publication in the Pacific Journal of Mathematics should be in typed form or offset-reproduced, double spaced with large margins. Underline Greek letters in red, German in green, and script in blue. The first paragraph or two must be capable of being used separately as a synopsis of the entire paper. It should not contain references to the bibliography. Manuscripts, in duplicate if possible, may be sent to any one of the four editors. Please classify according to the scheme of Math. Rev. 36, 1539-1546. All other communications to the editors should be addressed to the managing editor, Richard Arens, University of California, Los Angeles, California, 90024.

50 reprints are provided free for each article; additional copies may be obtained at cost in multiples of 50 .

The Pacific Journal of Mathematics is published monthly. Effective with Volume 16 the price per volume (3 numbers) is $\$ 8.00$; single issues, $\$ 3.00$. Special price for current issues to individual faculty members of supporting institutions and to individual members of the American Mathematical Society: $\$ 4.00$ per volume; single issues $\$ 1.50$. Back numbers are available.

Subscriptions, orders for back numbers, and changes of address should be sent to Pacific Journal of Mathematics, 103 Highland Boulevard, Berkeley, California, 94708.

PUBLISHED BY PACIFIC JOURNAL OF MATHEMATICS, A NON-PROFIT CORPORATION

Printed at Kokusai Bunken Insatsusha (International Academic Printing Co., Ltd.), 7-17, Fujimi 2-chome, Chiyoda-ku, Tokyo, Japan.

* Acting Managing Editor. 


\section{Pacific Journal of Mathematics}

\section{Vol. 32, No. 3 \\ March, 1970}

Shair Ahmad, Dynamical systems of characteristic $0^{+} \ldots \ldots \ldots \ldots \ldots$

Charles A. Akemann and Bernard Russo, Geometry of the unit sphere of a $C^{*}$-algebra and its dual............................ 575

Philip Bacon, The compactness of countably compact spaces ......... 587

Richard Blaine Barrar and Henry Loeb, On the continuity of the nonlinear Tschebyscheff operator ............................ 593

L. Carlitz, Factorization of a special polynomial over a finite field ....... 603

Joe Ebeling Cude, Compact integral domains .................... 615

Frank Rimi DeMeyer, On automorphisms of separable algebras. II . . . . . 621

James B. Derr, Generalized Sylow tower groups .................. 633

Raouf Doss, Some inclusions in multipliers ................... 643

Mary Rodriguez Embry, The numerical range of an operator........... 647

John Froese, Domain-perturbed problems for ordinary linear differential

operators..................................... 651

Zdeněk Frolík, Absolute Borel and Souslin sets ..................... 663

Ronald Owen Fulp, Tensor and torsion products of semigroups .......... 685

George Grätzer and J. Płonka, On the number of polynomials of an

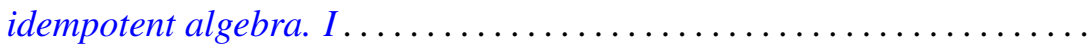

Newcomb Greenleaf and Walter Read, Positive holomorphic differentials on

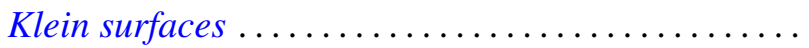

John Willard Heidel, Uniqueness, continuation, and nonoscillation for a second order nonlinear differential equation ................. 715

Leon A. Henkin, Extending Boolean operations................... 723

R. Hirshon, On hopfian groups .......................... 753

Melvin Hochster, Totally integrally closed rings and extremal spaces ..... 767

R. Mohanty and B. K. Ray, On the convergence of a trigonometric integral ..................................

Michael Rich, On a class of nodal algebras .................... 787

Emile B. Roth, Conjugate space representations of Banach spaces ........ 793

Rolf Schneider, On the projections of a convex polytope .............. 799

Bertram Manuel Schreiber, On the coset ring and strong Ditkin sets ...... 805

Edgar Lee Stout, Some remarks on varieties in polydiscs and bounded

holomorphic functions .........................

James Edward Ward, Two-groups and Jordan algebras . 